\title{
Dynamical Modeling of the Nuclear Fission Process at Low Excitation Energies
}

\author{
I. I. Gontchar ${ }^{1}$, M. V. Chushnyakova1,2, E. P. Oskin'1, E. G. Demina1 \\ ${ }^{1}$ Physics and Chemistry Department, Omsk State Transport University, Omsk, Russia \\ ${ }^{2}$ Physics Department, Omsk State Technical University, Omsk, Russia \\ Email: vigichar@hotmail.com
}

Received December 2013

\section{Abstract}

Two recipes for modeling the dynamics of the nuclear fission process are known in literature. The underlying equations contain the driving, dissipative, and random forces. The two recipes are mostly different in the prescriptions for the driving force. In this work we carefully compare these driving forces and the resulting fission rates. It turns out that the rates may be very close or strongly different depending on the value the shell correction to the nuclear deformation energy. We give arguments in favor of one of the recipes.

\section{Keywords}

Nuclear Fission, Dynamical Modeling, Stochastic Differential Equations, Fermi Gas Model

\section{Introduction}

Numerous experiments related to the nuclear fission process [1,2], in particular those aiming for discovering new superheavy elements [3-5], require practical and reliable numerical modeling of fission dynamics at low excitation energies. The most important physical quantity characterizing the fission process, although not observable, is the fission rate which is by definition

$$
\begin{gathered}
R\left(t_{i}\right)=\frac{1}{N_{\text {tot }}-N_{f}\left(t_{i}\right)} \frac{N_{f}\left(t_{i}\right)-N_{f}\left(t_{i-1}\right)}{\Delta t}, \\
N_{f}\left(t_{i}\right)=\sum_{j=1}^{i} \Delta N_{f}\left(t_{j}\right) .
\end{gathered}
$$

Here $\Delta N_{f}\left(t_{j}\right)$ is the number of trajectories reached the scission point during the time interval $\Delta t=t_{j}-t_{j-1}$; $N_{f}\left(t_{i}\right)$ is the number of trajectories reached the scission point until the time moment $t_{i}$.

Presently the dynamical modeling of fission is mostly performed by solving numerically the stochastic differrential equations with the white noise. In the simplest one dimensional case these equations read

$$
\begin{gathered}
d q=p m^{-1} d t \\
d p=-\eta m^{-1} p d t+f d t+\sqrt{2 \eta T} \cdot d W
\end{gathered}
$$


Here $d t$ is the time interval during which the collective coordinate (deformation parameter) $q$ and its conjugate momentum $p$ increase by $d q$ and $d p$, respectively; $W$ is the Wiener process whose increment $d W$ obeys the normal distribution with the variance $d t(d W \sim \sqrt{d t}) ; \eta$ and $m$ are the friction and inertia parameters, respectively. For the sake of simplicity we consider $\eta$ and $m$ to be deformation independent $(\beta=\eta / m)$.

The driving force can be calculated by two methods. The first one is a generalization [6] of the thermodynamical approach, which was developed for the one dimensional problem in $[7,8]$ and later was extended to the multidimensional case in [9]. Note that this method is applied, except [6], for the high excitation energies when the shell correction to the nuclear Potential Energy $(V, \mathrm{PE})$ and to the single particle Level Density Parameter ( $a, \mathrm{LDP}$ ) is believed does not play a role. The driving force calculated using this approach, $f_{I}$, is defined as a proper derivative of a thermodynamical potential:

$$
f_{I}=-\left(\frac{\partial F}{\partial q}\right)_{T}=T\left(\frac{\partial S}{\partial q}\right)_{E}
$$

is the Helmholtz free energy, $S(q, E)$ is the nucleus entropy, and $E$ is its total excitation energy. Within the framework of the Fermi gas model, the entropy reads

$$
S(q, E)=2[a(q, U) U(q)]^{1 / 2}
$$

The intrinsic excitation energy $U$ is related to $E$ and $P E$ as follows:

$$
U(q)=E-V(q)=E-V_{L}(q)-V_{S}(q)
$$

Here $V_{L}(q)$ and $V_{S}(q)$ are the smooth (liquid drop) part of PE and the shell correction, respectively.

According to [10] the LDP can be written as

$$
a(q, U)=a_{L}(q)\left\{1+g(U) V_{s}(q) U^{-1}\right\}
$$

The function $g(U)$ reaches unity when $U$ becomes large enough:

$$
g(U)=1-\exp (-k U)
$$

$k=0.054 \mathrm{MeV}^{-1}$. The deformation dependence of the smooth part of the LDP is defined as follows [10]: $a_{L}(q)=a_{1} A+a_{2} A^{2 / 3} B_{s}(q)$. Here $A$ is the nucleus mass number, $a_{1}=0.073 \mathrm{MeV}^{-1}$ and $a_{2}=0.095 \mathrm{MeV}^{-1}, B_{s}(q)$ is the dimensionless nuclear surface area.

Using Equations (5), (7) and (8) we obtain for the driving force the following expression

$$
f_{I}=-V_{L}^{\prime}-V_{S}^{\prime}+a_{E}^{\prime} T^{2}
$$

The prime denotes the derivative with respect to the coordinate, the subscript " $E$ " indicates that the derivative must be taken keeping $E$ constant.

Within the framework of the second approach $[11,12]$ the driving force $f_{\text {II }}$ is calculated according the formula, which (using our notations) reads

$$
f_{I I}=-V_{L}^{\prime}-(1-g) V_{S}^{\prime}
$$

Equations (10) and (11) look very much different. One hardly can expect to find the fission rates similar if $f_{I}$ and $f_{I I}$ are used in Equation (4). In order to check whether the rates are different indeed we construct a schematic PE which reproduces the main distinct features of the PE of ${ }^{236} \mathrm{U}$-nucleus. This schematic $V(q)$ is shown in Figure 1 along with $V_{L}(q)$ and $V_{S}(q)$.

One sees clearly the double humped structure of the fission barrier which is well known for the actinide nuclei [13].

We modeled the fission process applying Equations (3), (4) and the driving forces $f_{I}$ and $f_{I I}$. The PE of Figure 1 was used for the modeling. The initial conditions corresponded to the Brownian particles at rest at the left minimum of the PE. Typical fission rates resulted from this modeling according to Equation (1) are shown in Figure 2. Although the quasistationary rate $R_{\mathrm{DII}}$ exceeds $R_{\mathrm{DI}}$ by some $20 \%$, one can expect much larger difference considering Equations (10) and (11).

In order to figure out the reason for this surprisingly small difference let us transform Equation (10). The derivative of the LDP reads: 


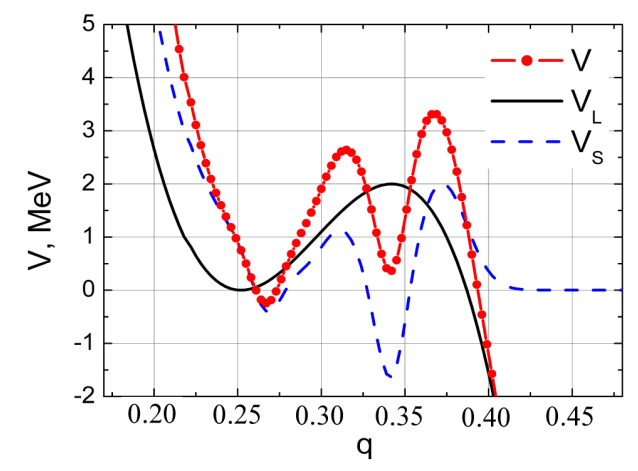

Figure 1. The schematic PE for ${ }^{236} U$ (curve with dots) as a function of collective coordinate along with its components: the smooth (solid curve) and shell correction (dashed curve) parts.

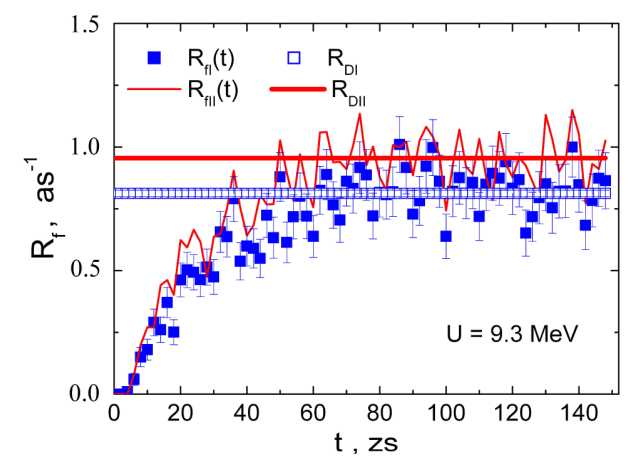

Figure 2. The dynamical fission rates calculated for ${ }^{236} \mathrm{U}$ according to Equation (1) versus modeling time. The horizontal lines indicate the quasistationary values of the rates.

$$
\left(\frac{\partial a}{\partial q}\right)_{E}=a_{L}\left[\frac{g^{\prime} \cdot V_{S}}{U}+\frac{g \cdot V_{S}^{\prime}}{U}-\frac{g \cdot V_{S} \cdot U^{\prime}}{U^{2}}\right]+a_{L}^{\prime} \frac{g \cdot V_{S}}{U}
$$

Keeping in mind that $U^{\prime}=-V_{L}^{\prime}-V_{S}^{\prime}$ and $g^{\prime}=k U^{\prime} \exp (-k U)$ we convert Equation (10) to the form

$$
f_{I}=-V^{\prime}+a_{L}^{\prime} \varepsilon+\frac{U}{1+\varepsilon}\left[\frac{V_{S} k V^{\prime}}{U}-\varepsilon k V^{\prime}+\frac{g \cdot V_{S}^{\prime}}{U}-\frac{\varepsilon V^{\prime}}{U}\right]
$$

Here $\varepsilon=g(U) V_{S}(q) U^{-1}$ turns out to be a small parameter not exceeding 0.1 in the region of the barrier at $U>10 \mathrm{MeV}$ in particular because in the considered case $V_{s}(q)$ is in order of $2 \mathrm{MeV}$. Omitting in (13) the terms obviously proportional to $\varepsilon$ we arrive at the formula

$$
f_{I}=f_{I I}+V_{S} k V_{L}^{\prime}+V_{S} k V_{S}^{\prime}+g \cdot V_{L}^{\prime}
$$

Since $k^{-1}$ is about $20 \mathrm{MeV}$, in Equation (14) the terms containing $k V_{S}(q)$ are rather small. The last term is small because $V_{L}(q)$ is rather smooth (see Figure 1). We believe that this derivation explains relatively small difference between $f_{I}$ and $f_{I I}$ resulting in small difference between the corresponding fission rates in Figure 2 .

We also have considered the case of a nucleus with the large shell correction at the ground state, namely the double magic lead-208. The PE of Figure 3 was used for the modeling in this case. The absolute value of the shell correction is now about $12 \mathrm{MeV}$. The way of modeling Figure 4. One sees that in this case $R_{D I I}$ exceeds $R_{D I}$ by factor of 2 whereas the conventional value of the intrinsic excitation energy at which the shell correction ceases to manifest itself (and consequently $R_{\mathrm{DII}}$ and $R_{\mathrm{DI}}$ must converge) is about $60 \mathrm{MeV}$.

It is worthwhile to discuss and illustrate what does the smearing of the shell correction exactly mean. Within the framework of the first recipe, it turns out that one can calculate the fission rate ignoring completely the shell 
correction in the PE (i.e. using $V_{L}$ instead of $V$ in Equation (7)) and in the LDP (i.e. using $a_{L}$ instead of $a$ in Equation (6)). The corresponding fission rate $R_{D I L}$ must converge with $R_{D I}$ as $E$ increases. This is seen in Figure 5 where the quasistationary rates resulting from dynamical modeling are shown. In order to quantify the convergence between $R_{D I L}$ and $R_{D I}$ we have calculated the fractional difference $R_{D I} / R_{D I L}-1$. It has turned out that the difference goes to zero reaching circa $-10 \%$ at $E=60 \mathrm{MeV}$ as it is known for the statistical rates.

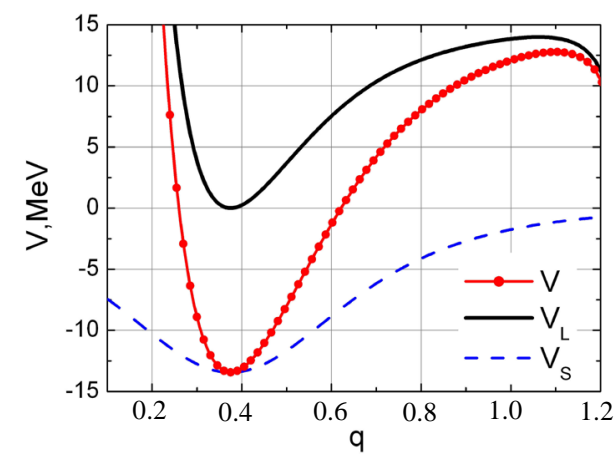

Figure 3. The schematic $\mathrm{PE}$ for ${ }^{208} \mathrm{~Pb}$ (curve with dots) as a function of collective coordinate along with its components: the smooth (solid curve) and shell correction (dashed curve) parts.

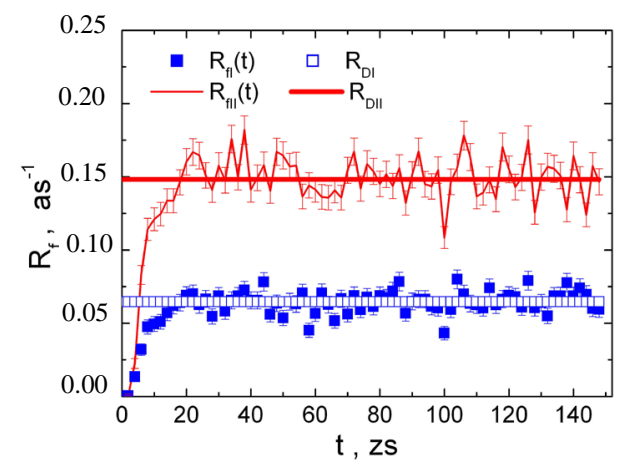

Figure 4. The dynamical fission rates calculated for ${ }^{208} \mathrm{~Pb}$ according to Equation (1) versus modeling time. The horizontal lines indicate the quasistationary values of the rates.

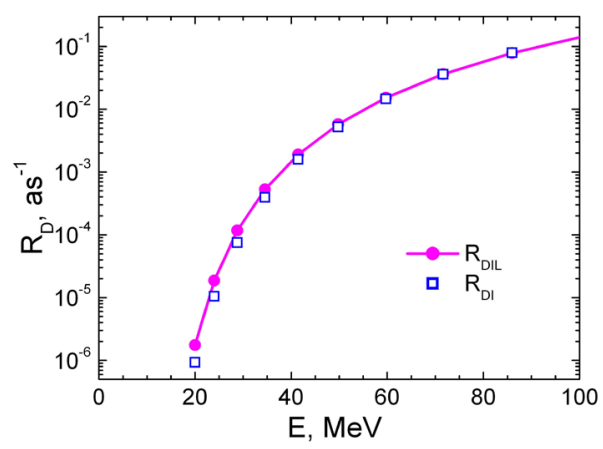

Figure 5. The dynamical quasistationary fission rates calculated for ${ }^{208} \mathrm{~Pb}$ nucleus accounting for the shell correction (squares) and ignoring those (line with circles). 
Within the framework of the second recipe, the convergence is not reached because the reference point for the excitation energy always include the potential energy with the shell correction (see Equation (6) of [12]).

To finalize, we have compared two approaches for calculating the driving force for the nuclear fission process at low excitation energies when the shell effects are expected to be significant. We have found that in the case of uranium-236 nucleus the quasistationary decay rates $R_{D I}$ and $R_{D I I}$ resulting from these approaches are rather close (the difference is about 20\%). This is however just because for this nucleus the shell correction is small in comparison with the typical energy $k^{-1}=18.5 \mathrm{MeV}$ controlling the smearing out the shell effects. For the lead208 nucleus with larger value of the shell correction, the difference between $R_{D I}$ and $R_{D I I}$ reaches factor of 2 . This is significantly larger than the difference between the rates calculated within the frame work of the first approach with and without the shell correction. Since the first approach is based on the thermodynamical arguments, we are inclined to make favor to it in comparison with the second one.

\section{Acknowledgements}

M. V. C. and E. G. D. are grateful to the Dmitry Zimin Foundation 'Dynasty’ for financial support.

\section{References}

[1] Wagemans, C. (1991) The Nuclear Fission Process. CRC Press Inc.

[2] Hilscher, D. and Rossner, H. (1992) Dynamics of Nuclear Fission. Annals of Physics (France), 17, 471-552. http://dx.doi.org/10.1051/anphys:01992001706047100

[3] Oganessian, Yu. (2013) Heaviest Nuclei. Nuclear Physics News, 23, 15-21. http://dx.doi.org/10.1080/10619127.2013.767694

[4] Itkis, I.M., et al. (2011) Fission and Quasifission Modes in Heavy-Ion-Induced Reactions Leading to the Formation of Hs. Physical Review C, 83, 064613. http://dx.doi.org/10.1103/PhysRevC.83.064613

[5] Oganessian, Yu.Ts., et al. (2010) Synthesis of a New Element with Atomic Number Z = 117. Physical Review Letters, 104, 142502. http://dx.doi.org/10.1103/PhysRevLett.104.142502

[6] Pavlova, E.G. and Gontchar, I.I. (2012) Dissipative Statistical and Dynamical Fission Rates: Case of the Microcanonical Ensemble. Proceedings of the 4th International Conference of the Current Problems in Nuclear Physics and Atomic Energy, Kyiv, 3-7 September 2012, 315-319.

[7] Gontchar, I.I., Fröbrich, P. and Pischasov, N.I. (1993) Consistent Dynamical and Statistical Description of Fission of Hot Nuclei. Physical Review C, 47, 2228-2235. http://dx.doi.org/10.1103/PhysRevC.47.2228

[8] Gontchar, I.I. and Fröbrich, P. (1993) Nuclear Fission: Combining the Dynamical Langevin Equation with the Statistical Model. Nuclear Physics A, 551, 495-507. http://dx.doi.org/10.1016/0375-9474(93)90459-B

[9] Adeev, G.D., Karpov, A.V., Nadtochy, P.N. and Vanin, D.V. (2005) Multidimensional Stochastic Description of the Excited Nuclei Fission Dynamics. Physics of Particles and Nuclei, 36, 733-820.

[10] Ignatyuk, A.V., Itkis, M.G., Okolovich, V.N., Smirenkin, G.N. and Tishin, A.S. (1975) Fission of Pre-Actinide Nuclei. Excitation Functions for the ( $\alpha$, f) Reaction. Physics of Atomic Nuclei (Yadernaya Fizika), 21, 1185-1205.

[11] Aritomo, Y. and Ohta, M. (2004) Dynamical Calculation for Fusion-Fission Probability in Superheavy Mass Region, Where Mass Symmetric Fission Events Originate. Nuclear Physics A, 744, 3-14. http://dx.doi.org/10.1016/j.nuclphysa.2004.08.009

[12] Aritomo, Y. and Chiba, S. (2013) Fission Process of Nuclei at Low Excitation Energies with a Langevin Approach. Physical Review C, 88, 044614. http://dx.doi.org/10.1103/PhysRevC.88.044614

[13] Brack, M., Damgaard, J., Jensen, A.S., Pauli, H.C., Strutinsky, V.M. and Wong, C.Y. (1972) Funny Hills: The ShellCorrection Approach to Nuclear Sell Effects and Its Applications to the Fission Process. Reviews of Modern Physics, 44, 320-405. 